\title{
FEDEP V1.4: AN UPDATE TO THE HLA PROCESS MODEL
}

\author{
Robert Lutz \\ Johns Hopkins University \\ Applied Physics Laboratory \\ Laurel, MD 20723, U.S.A.
}

\begin{abstract}
The Department of Defense (DoD) High Level Architecture (HLA) for modeling and simulation (M\&S) was developed as a means of facilitating interoperability among simulations and promoting reuse of simulations and their components. Although the three HLA specifications together provide the necessary technical foundation for developing distributed, interoperable simulation applications, they do not explicitly define how such applications are developed. In this regard, in order to provide practical guidance to the HLA user community, the Defense Modeling and Simulation Office (DMSO) has sponsored the creation of a process model that describes a structured, common sense approach to HLA federation development. This process model is known as the HLA Federation Development and Execution Process (FEDEP) Model.

The primary purpose of this paper is to outline the modifications that have been incorporated in transitioning from FEDEP V1.3 to FEDEP V1.4. This paper will also describe the Concept of Operations (ConOps) for how new releases of the FEDEP are produced, and will identify other related products which may be used in concert with the FEDEP to support the needs of HLA federation developers.
\end{abstract}

\section{BACKGROUND}

The Department of Defense (DoD) High Level Architecture (HLA) has been developed in response to the DoD Modeling and Simulation (M\&S) Master Plan, which calls for a DoD-wide common technical framework which will apply to the full range of potential $M \& S$ applications. The objective of the HLA is to facilitate interoperability among simulations and promote reuse of simulations and their components (DoD 5000.59-P 1995).

Early in the development of the HLA, a strategy was formulated by which the initial HLA specifications (HLA Rules, Interface Specification, and Object Model Template) would be utilized and tested in each of four different prototype federations (protofederations). These protofederations would then provide the feedback necessary to fully mature the architecture. This approach was implemented early in 1995, and provided the basic mechanism for HLA evolution until the release of the HLA baseline in August 1996.

During this prototyping phase, the protofederations identified a critical need for guidance regarding the process of building HLA federations. That is, since no generalized view of the HLA federation development process existed at that time, each protofederation was required to define their own development procedures and practices. This required a high degree of experimentation by trial and error, and generally resulted in a relatively high (and inefficient) level of resource consumption. The outcome of this experience was a strong recommendation to the HLA program to develop a common process model for HLA applications.

The first release of the HLA Federation Development and Execution Process (FEDEP) model occurred in August, 1996. The development of this process model represented a coordinated effort between the HLA technical support team and representatives of the HLA protofederations. To facilitate this collaboration, the HLA OMT Working Group provided an open forum for each of the protofederations to share their individual approaches (and resulting experiences) regarding federation development methodologies and procedures. It was then through open discussion of alternative federation development and execution strategies within the working group that consensus was reached regarding "best practices" for a generalized process model which could potentially support all HLA user communities (Lutz 1998).

Since the original release of the FEDEP, the FEDEP has continued to evolve and mature based on feedback from the HLA user community. In Section 3, this paper describes the process by which this evolution has occurred.

\section{PURPOSE/DESCRIPTION}

The primary purpose of the FEDEP is to explicitly identify and describe the activities necessary to build HLA federations. Such activities are typically generic in nature, and collectively define a straightforward, common sense systems engineering approach to the development of 
distributed systems. Besides defining a viable and useful process framework for HLA federation developers, the FEDEP also:

- Provides a common reference for facilitating communication between federation development team members

- Quickly orients new users to basic HLA principles

- Establishes a common foundation for defining function-specific views of the federation development process

- Provides an organizing mechanism for sharing federation development experiences at the semi-annual Simulation Interoperability Workshops (SIW) in Orlando Florida

The FEDEP defines the HLA federation development process at two different levels of abstraction (DMSO 1999). In the top-level view, the process is defined by the six major development steps identified in Figure 1. In the more detailed view, each of these six steps are decomposed into a set of lower-level component activities, where activity relationships and the flow of data between activities are explicitly defined. Two very important, fundamental principles that underlie both of these views are as follows:

- The FEDEP is not prescriptive, in that it does not specify a "one size fits all" process for all
HLA users. Rather, the FEDEP must generally be tailored to meet the unique needs and requirements of each new application. For instance, in large complex federations with many distributed team members, a highly structured, formalized development process is commonly needed to maintain adequate control and coordination across the project. However, in less complex applications supported by smaller, tightly knit federation development teams, far less formalization may be needed. Federation resource limitations and the availability of reusable federation products may also significantly affect the development process chosen for a specific application.

- The FEDEP does not replace the native, lowlevel systems engineering methodologies of individual user communities. Rather, the FEDEP is intended as a high-level framework for identifying required federation development activities, and how those activities interrelate within the overall federation development process. The specific nature and sequencing of these activities are entirely at the discretion of the federation development team, and are frequently shaped by the local practices and procedures of the responsible organizations.

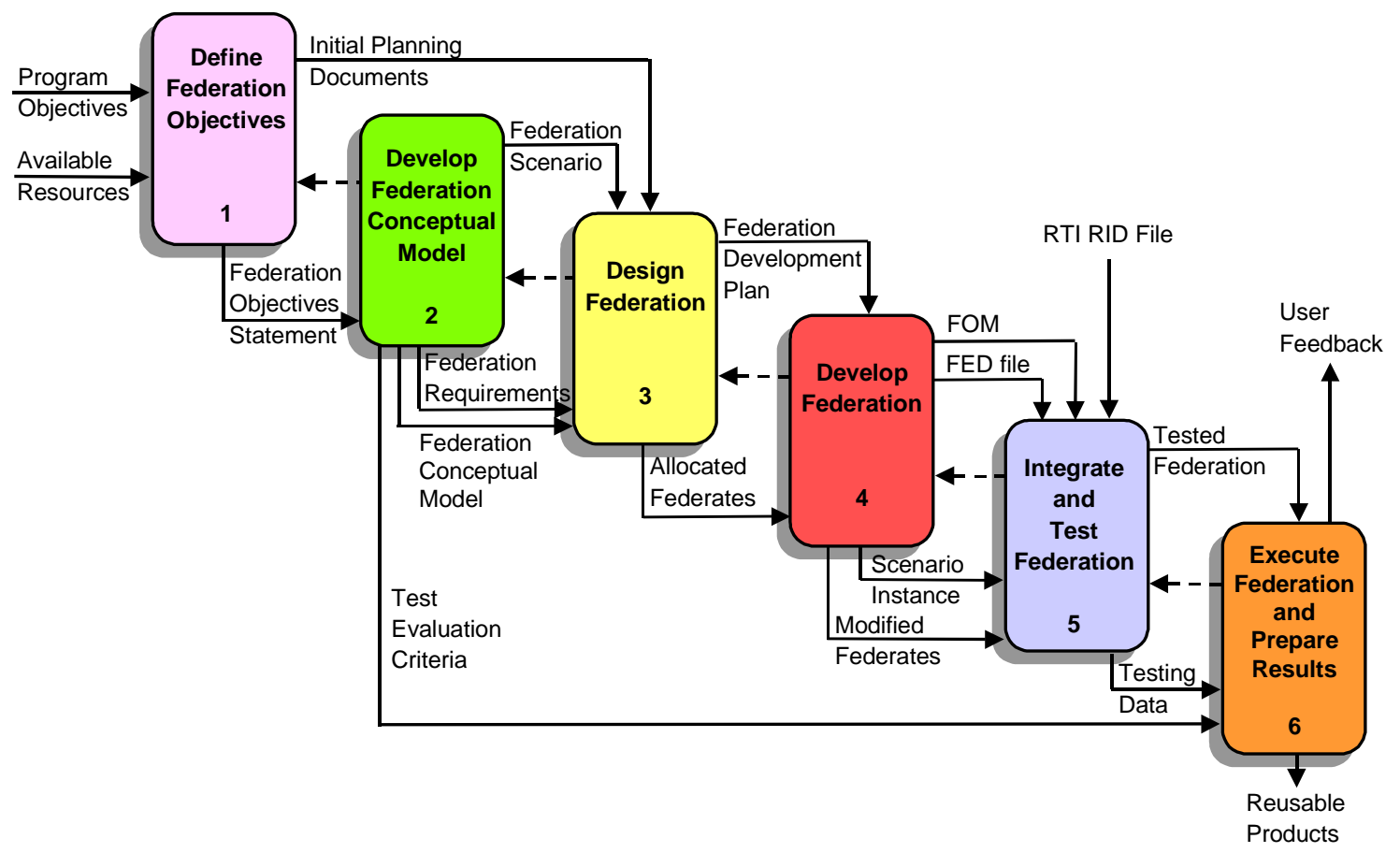

Figure 1: Six Step Federation Development Process 


\section{CONOPS}

In order to facilitate the controlled evolution of the FEDEP document, a structured Concept of Operations (ConOps) was established in early 1997. This ConOps is illustrated in Figure 2, and is defined by the steps described below:

1. As new programs are established, strongly encourage the use of the FEDEP as a starting framework for the development of the federation.

2. During the development of the federation, encourage programs to identify and document any potential improvements to the FEDEP description. Request that these suggestions be submitted via the HLA home page, and/or documented (along with a case history of their federation development) in the form of abstracts and technical papers for consideration by the Simulation Interoperability Standards Organization (SISO).

3. Utilize the SISO Federation Development Process (PROC) Forum within the Simulation Interoperability Workshops as a mechanism for open sharing of federation development experiences and for open discussion of FEDEP change proposals.

4. Conduct a technical exchange with representatives of HLA Architecture Management Group (AMG) members to review change proposals received from the SIW, change proposals received via the HLA home page, and change proposals submitted directly by technical exchange participants. Discuss the relative merits of each proposed modification.

5. Prepare a listing of those FEDEP change proposals for which consensus was reached within the technical exchange.

6. Conduct an internal HLA program review of the listing to ensure that there are no conflicts or inconsistencies between the FEDEP description and other related HLA activities.

7. Prepare a draft version of the FEDEP document that incorporates all approved changes. Provide the draft document to the AMG for review. Incorporate all AMG feedback as appropriate, and post the finalized version of the FEDEP document on the HLA home page.

To date, there have been four complete cycles of this ConOps, resulting in the release of four new revisions of the FEDEP document (V1.1-V1.4). Each of these revisions has occurred at approximately six month intervals. The next section of this paper will focus on the changes that were identified and implemented in the most recent cycle of the FEDEP ConOps.

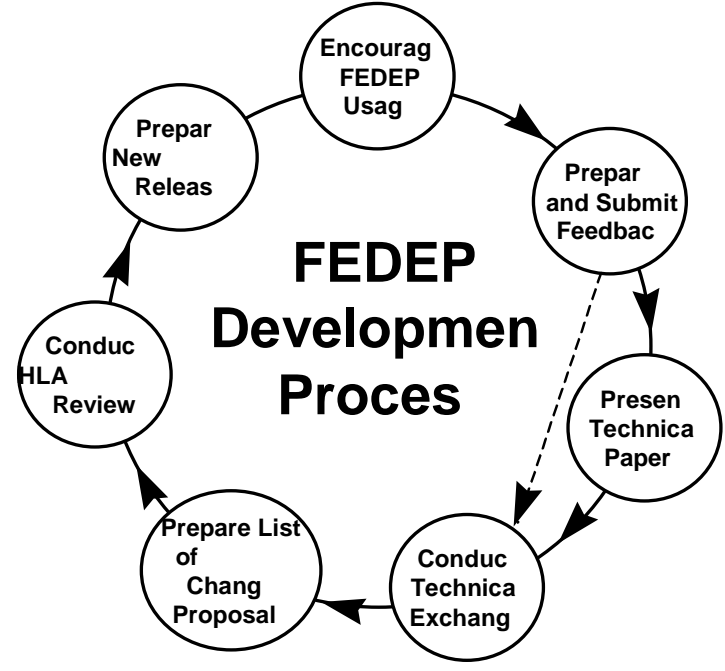

Figure 2: FEDEP Concept of Operations

\section{FEDEP V1.4 DEVELOPMENT}

The SIW that provided the main source of user feedback on the FEDEP V1.3 document occurred on 15-19 March 1999 (SISO 1999). Per the ConOps described above, a technical exchange was held on 8 April to review the inputs from the SIW, and allow AMG representatives to provide direct input on the content and direction of the FEDEP document. Based on the technical discussions that occurred within that forum, a favorable consensus was achieved on a number of the proposed modifications. Two of these proposed modifications represented relatively major changes to the FEDEP document, and are summarized below:

- Create an executive summary. In previous versions of the FEDEP, there was no executive summary included in the document. This was identified as a deficiency in the past, as readers were forced to read through much of the document before they could gain a basic understanding of its intended usage. In particular, the document needs a clear, concise executive summary that would 1) define the purpose of the FEDEP, 2) identify the potential customers of the FEDEP, 3) delineate the potential value of the FEDEP to these customers (i.e., why should they care?), and 4) describe the types of factors that will shape how the FEDEP is implemented for any particular application. By providing this information at the beginning of the FEDEP document, the reader will have the "big picture" of the fundamental needs that the FEDEP is intending to address before the process model description itself is provided. 
- $\quad$ Partition the middle phase of the FEDEP into two distinct steps. One of the original objectives of the FEDEP was to ensure a uniform treatment of the major development activities identified throughout the process description. However, in FEDEP V1.3, there were many more activities and tasks identified in the third step of the process model (Federation Design and Development) than in any other of the major steps. In order to improve the uniformity by which the major steps of the FEDEP are described, this middle step needs to be partitioned into two distinct steps, that of Design Federation and Develop Federation. Although no new content is required, this restructuring will allow the tasks and activities that comprise these major steps to become clearer and more visible to the user.

In addition to these proposals, several other minor modifications were proposed for which there was broad agreement across the participants at the technical exchange:

- Recognize the need for establishing certain operating agreements among federation participants. Examples include agreements on initialization procedures, synchronization points, and save/restore policies.

- Recognize that, in certain circumstances, HLA applications may have needs for federation products beyond those explicitly referenced in the FEDEP.
- Recognize the need for consistent representations of shared data and algorithms among federation members (e.g. terrain data, atmospheric data, etc.).

This listing of proposed FEDEP modifications was reviewed and approved by the HLA program, and was incorporated into a draft version of FEDEP V1.4 on 23 April for review by the HLA AMG. During development of this draft, each of the two new partitions of the previous Federation Design and Development step was decomposed into its lower-level component activities. An illustration of this new decomposition is shown in Figure 3. The Design Federation step is described according to three main activities:

- Select Federates: Determine the suitability of individual simulation systems to become members of the federation. Although normally driven be the perceived ability of potential federation members to represent entities and events in the domain of interest, additional technical (e.g., scalability, portability) and programmatic (e.g., availability, verification/validation history) selection criteria may also apply.

- Allocate Functionality: Perform an allocation of required federation functionality to the selected federates. Conduct federation design tradeoff investigations as necessary.

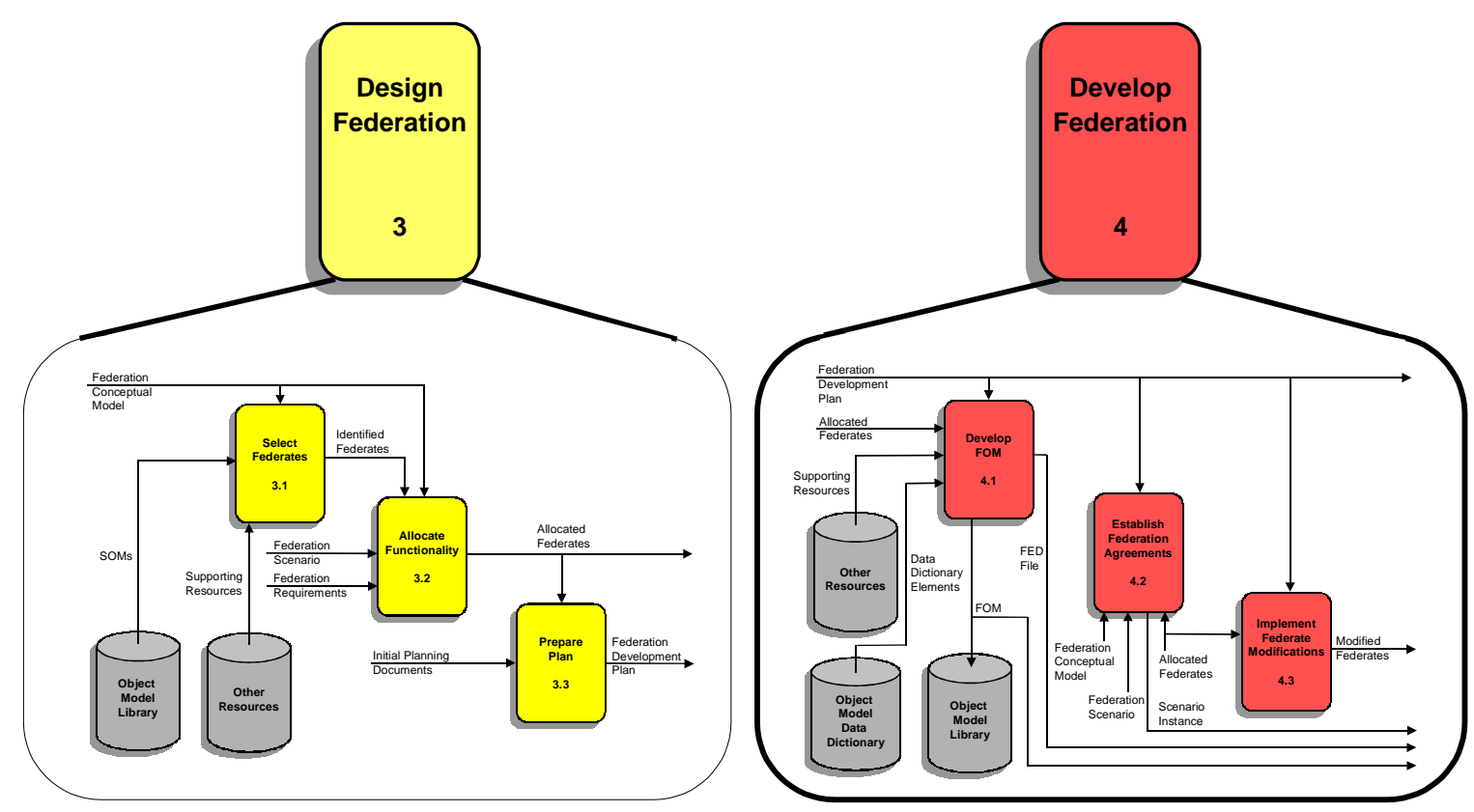

Figure 3: Decomposition of "Design Federation" and "Develop Federation" 
- Prepare Plan: Create a coordinated plan to guide the development, integration, test, and execution of the federation. This includes the fundamental systems engineering methodology that will be used across the federation, the tools that will be used, and assignments of responsibility for federation products (and required delivery dates) to the federates.

The Develop Federation step is also described according to three main activities:

- Develop FOM: Identify an overall strategy for collaborative development of the Federation Object Model (FOM), and implement that strategy using all applicable automated tools.

- Establish Federation Agreements: Identify and then collaborate (among federates) on the resolution of pertinent interoperability issues, such as shared data/algorithm consistency and federation time management.

- Implement Federate Modifications: Implement all required federate software modifications. These modifications may represent changes or extensions to the domain-specific functionality provided by the federate, or may be changes or extensions to the federate's HLA interface.

Subsequent to completion of the HLA AMG review, the finalized version of FEDEP V1.4 was posted on the HLA home page on 9 June. This version of the FEDEP is intended to support the process needs of the HLA community until the next full cycle of the ConOps is completed later this calendar year.

\section{RELATED ACTIVITIES AND PRODUCTS}

In addition to the recent development of FEDEP V1.4, several related efforts have also resulted in the development of supporting products for HLA federation developers. A brief description of each of these products is provided here:

FEDEP Checklists: A checklist of low-level development tasks that together provide the next layer of detail for guiding HLA users through the steps of the FEDEP. While the HLA FEDEP document describes what the FEDEP is, the checklists explain how to do it. The goal is to guide an uninitiated HLA user through the steps necessary to produce a working federation that meets his or her requirements (Lutz, Salisbury, and Turrell 1999).

The checklists are organized along the six steps of the FEDEP model, with an individual checklist for each step. Each checklist contains two classes of directed activities: technical and managerial. The items in each checklist can be categorized as:

- Decisions that need to be made

- Factors that need to be considered and evaluated

- Actions that need to be completed before moving on

- Issues that must be coordinated between the various members of the project

- Documents that should be prepared

The initial draft of the FEDEP Checklists was primarily based on the federation development experiences of a selected set of HLA programs. This draft was then reviewed by appropriate representatives of AMG programs, and feedback incorporated into a finalized version (V1.4) of the initial release. This release was posted on the HLA home page in July. Future releases of the FEDEP Checklists will be driven by the same ConOps that is currently the basis for FEDEP development.

FEDEP Technical Library: This product provides a library of FEDEP-related technical papers selected from various simulation conferences and workshops. Access to this technical library is through the HLA home page. Several of the papers in the library represent case studies on the use of the FEDEP in support of applications in different user communities. However, some of these papers also represent "overlays" on the FEDEP in such areas as security and VV\&A. Although the FEDEP Technical Library is presently limited in size, it is expected that this repository of federation development knowledge will continue to grow as more and more programs transition to the HLA.

FEDEP Clickable Image: This utility allows users to view any of the graphical images provided in the FEDEP document directly on their computer screen. Then, by simply moving the mouse to any activity box shown on their display, users may immediately view a textual description of that activity. This utility is primarily geared toward new users of the HLA, although it also provides a quick process model reference to more experienced users.

There also continues to be significant progress on the development of automated tools to support the FEDEP. Although a complete description of the many government and commercial tools that are currently available to support federation development activities is beyond the scope of this paper, the DMSO-sponsored HLA Tools Bulletin Board provides a brief, non-commercial, description of a wide variety of HLA tools and services, along with appropriate point of contact information. The Bulletin Board is offered as a service to the community to inform personnel about the tools available to support their transition to the HLA. 


\section{SUMMARY}

This paper was intended to highlight recent activities related to FEDEP development. The ConOps described in this paper provides a viable framework for structured, controlled evolution of the FEDEP document. The fourth major cycle of this ConOps has resulted in the identification and incorporation of several new process model improvements. In addition, several supporting products have been recently developed to provide additional assistance and guidance to FEDEP users.

The HLA home page may be found at http://hla.dmso.mil/. Through this page, any of the products discussed in this paper may be accessed. In general, the most effective way for interested parties to stay informed about new developments with respect to HLA processes and supporting tools is through subscription to the HLA Online email reflector. A one line email containing "SUBSCRIBE HLA_ONLINE your-firstname your-last-name” to listproc@msis.dmso.mil will establish your subscription.

\section{REFERENCES}

Under Secretary of Defense for Acquisition and Technology, "Department of Defense Modeling and Simulation Master Plan, DoD 5000.59-P," October 1995.

Lutz R., "FEDEP V1.1", Simulation Interoperability Workshop, 98S-SIW-236, March 1998.

Defense Modeling and Simulation Office (DMSO), "HLA Federation Development and Execution Process (FEDEP) Model, Version 1.4,” June 1999.

Simulation Interoperability Standards Organization (SISO), "Spring 1999 Simulation Interoperability Workshop Proceedings", http://siso.sc.ist.ucf.edu/siw/99Spring/ view-papers.htm, March 1999.

Lutz R., Salisbury M., Turrell, C., "HLA Federation Development and Execution Process (FEDEP) and Supporting Tools", 1999 Summer Computer Simulation Conference, July 1999.

\section{AUTHOR BIOGRAPHY}

ROBERT LUTZ is a principal scientist at the Johns Hopkins University Applied Physics Laboratory. He has over 18 years of experience in simulation design and development. He is currently leading or participating in several HLA-related projects, including development of the HLA FEDEP. He is a member of the HLA technical support team, is the lead editor for IEEE Standard P1516.2 (Object Model Template), and serves as the chair of the SISO Federation Development Process Forum. 